\title{
Chuva de sementes de espécies lenhosas florestais em mosaicos de floresta com Araucária e campos no Sul do Brasil
}

\author{
Melina Marchesini Grassotti dos Santos ${ }^{1,3}$, Juliano Morales Oliveira ${ }^{2}$, Sandra Cristina Müller ${ }^{1}$ e Valério DePatta Pillar ${ }^{1}$
}

Recebido em 27/07/2010. Aceito em 27/01/2011

\begin{abstract}
RESUMO
(Chuva de Sementes de Espécies Lenhosas Florestais em Mosaicos de Floresta com Araucária e Campos no Sul do Brasil). Mecanismos de dispersão de diásporos e recrutamento de plântulas evitam a competição intraespecífica e possibilitam a colonização de novos ambientes. Em áreas de transição entre florestas e vegetação campestre esses processos não são aleatórios, e sim associados muitas vezes a indivíduos lenhosos isolados em tais áreas de campo ou a formações arbustivas. No sul do Brasil, as Florestas com Araucária formam mosaicos com áreas campestres e observa-se o avanço da floresta sobre os campos. O estudo teve como objetivo avaliar a chuva de sementes de espécies lenhosas florestais associadas a áreas de ecótonos de Floresta com Araucária e campos e a indivíduos lenhosos isolados na matriz campestre. Os estudos foram realizados no Centro de Pesquisas e Conservação da Natureza Pró-Mata PUCRS, em São Francisco de Paula, RS, em vegetação campestre excluída de fogo e de pastejo há 16 anos, circundada por florestas. A chuva de sementes foi avaliada mensalmente por um período de oito meses com o uso de coletores que foram posicionados em ecótonos de floresta e campos com diferentes fisionomias e sob indivíduos lenhosos isolados na matriz campestre. Tais ambientes foram comparados através de análise de variância univariada e multivariada para verificar padrões de chuva de sementes. Os resultados indicaram que a dispersão de sementes em áreas campestres ocorre preferencialmente associada a indivíduos isolados de Araucaria angustifolia e a formações arbustivas de Baccharis uncinella e que tais ambientes funcionariam como extensões de condições mais semelhantes à floresta na matriz campestre.
\end{abstract}

Palavras-chave: poleiros, formações arbustivas, Baccharis uncinella, zoocoria, facilitação

\begin{abstract}
(Seed rain of woody species in mosaics of Araucaria forest and grasslands in Southern Brazil). Mechanisms of seed dispersal and recruitment avoid intraspecific competition and allow plants to colonize new habitats. In transition areas between forest and grassland, these processes are not spatially random, but are often associated with isolated woody individuals in the grassland or with areas of continuous shrub cover. In southern Brazil, Araucaria forest forms a mosaic with grassland (Campos) and advance of forest over grassland is observed. This study aims to evaluate the pattern of diaspore dispersal of woody plants associated with ecotones between Araucaria forest and grassland and with isolated woody individuals in the grassland matrix. The study was carried out at the Centro de Pesquisas e Conservação da Natureza Pró-Mata PUCRS, in São Francisco de Paula, in grassland vegetation excluded from fire and grazing for 16 years, and which is surrounded by forest. Diaspore dispersal was evaluated for eight months by using collectors positioned in forest-grassland ecotones with different physiognomies and under isolated woody individuals in the grassland matrix. The different types of environment were compared by univariate and multivariate analysis of variance to verify seed-rain patterns. The results indicated that seed dispersal occurs preferentially associated with isolated woody individuals of Araucaria angustifolia and with continuous patches of Baccharis uncinella. We suggest that these habitats would function as extensions of more similar forest conditions within the grassland matrix.
\end{abstract}

Key words: perches, shrublands, Baccharis uncinella, zoochory, facilitation

\footnotetext{
Universidade Federal do Rio Grande do Sul, Departamento de Ecologia, Laboratório de Ecologia Quantitativa, Porto Alegre, RS, Brasil

2 Universidade do Vale do Rio dos Sinos, Programa de Pós-Graduação em Biologia, São Leopoldo, RS, Brasil

3 Autor para correspondência: melina.mgsantos@gmail.com
} 


\section{Introdução}

Estruturas proeminentes em áreas de campo como árvores isoladas, árvores mortas ainda em pé (Guevara et al. 1992; Peterson \& Haines 2000; Válio \& Scarpa 2001; Shiels \& Walker 2003; Clark et al. 2004; Gómes-Aparicio 2005) e até mesmo estruturas verticais artificiais (Holl 1998; Zanini \& Ganade 2005; Dos Santos \& Pillar 2007) mostram-se relevantes à atração de dipersores e ao incremento na chuva de sementes de espécies zoocóricas, facilitando os processos de sucessão florestal. A facilitação ocorre através de alterações micro-ambientais, como redução da radiação solar e evapotranspiração, mantendo assim condições de umidade e temperatura favoráveis ao recrutamento (Guevara et al. 1992; Callaway \& Walker 1997; Holmgren et al. 1997). O termo poleiros vem sendo usado para designar tais estruturas (Caldato et al. 1996; Nepstad et al. 1996; Holl 1998; Duarte et al. 2006a; Dos Santos \& Pillar 2007).

Formações arbustivas também são locais importantes para o recrutamento de sementes de espécies florestais quando comparadas a áreas predominantemente campestres (Fuentes et al. 1984). Assim como o recrutamento, espera-se que a chuva de sementes também ocorra de modo diferenciado sob tais formações. Portanto, a presença de associações arbustivas em áreas abertas, assim como árvores isoladas, podem ser consideradas facilitadoras de colonização e recrutamento de espécies florestais sobre áreas campestres. Estas estruturas de vegetação diferenciadas em áreas abertas parecem essenciais à atração de animais dispersores de espécies florestais e ao conseqüente processo de colonização, uma vez que estudos sobre padrões de distribuição de plântulas em áreas campestres sugerem que a dispersão zoocórica é de suma importância em áreas de expansão florestal na região (Duarte et al. 2006a, Duarte et al. 2006b, Fontoura et al. 2006; Zanini et al. 2006; Dos Santos \& Pillar 2007), assim como em outras regiões tropicais, já que a maioria das espécies arbóreas tropicais e subtropicais apresenta propágulos adaptados à dispersão por animais.

No planalto sul-brasileiro, a Floresta Ombrófila Mista forma mosaicos com formações campestres na região dos Campos de Cima da Serra (Rambo 1956; Klein 1960; Behling et al. 2004;) e, em áreas com exclusão de fogo e de pastejo, ocorrem processos de expansão florestal sobre áreas campestres junto a bordas de florestas contínuas ou de manchas florestais (capões) inseridas no campo (Pillar 2003; Machado 2004; Duarte et al. 2006b). Sob as mesmas condições, a expansão pode ser pela colonização aos saltos de indivíduos florestais na matriz campestre, como observado em locais sob indivíduos de Araucaria angustifolia (Bertol.) Kuntze (Araucariaceae) que se estabelecem no campo, facilitando assim o recrutamento de outras espécies florestais (Klein 1960; Duarte et al. 2006a). Os processos de expansão florestal sobre áreas de campo nesta região têm ocorrido em função das atuais condições climáticas, mais úmidas e quentes que no período de predomínio das formações campestres no sul do Brasil (Behling at al. 2004).

Nesta mesma região, observou-se também que o maior avanço da borda florestal ocorreu em áreas onde o campo apresentava fisionomia arbustiva alta caracterizada principalmente por indivíduos de Baccharis uncinella DC. (Asteraceae) (Oliveira \& Pillar 2004), arbusto predominante na fisionomia de várias regiões do planalto sul-brasileiro, principalmente em áreas campestres adjacentes às florestas, onde o campo deixa de ser manejado, como no Parque Nacional dos Aparados da Serra (obs. pessoal) e na Estação Ecológica de Aracuri (Waechter et al. 1984), sugerindo sua importância em processos de expansão florestal.

A avaliação desses processos, além de fornecer informações importantes para a compreensão da dinâmica natural de expansão florestal sobre campos, bem como à regeneração de florestas, tem implicações à conservação das formações campestres, uma vez que estas tendem a se modificar ao longo do tempo. Na região de estudo, estas formações são consideradas relictuais e mantenedoras de uma elevada e característica diversidade de espécies vegetais e animais, porém permanecem negligenciadas e não há práticas para sua conservação (Overbeck et al. 2007).

Este trabalho objetiva avaliar a colonização de diásporos de espécies arbóreas florestais em um mosaico de Floresta Ombrófila Mista e Campos no Rio Grande do Sul. Para tanto, são quantificadas a abundância de diásporos de espécies arbóreas florestais dispersados em áreas de transição floresta-campo (borda) e sob indivíduos de $B$. uncinella (arbusto de campo) e de A. angustifolia (árvore) isolados na matriz campestre, com vistas a inferir relações entre dispersão de diásporos e a dinâmica de expansão da floresta sobre os campos.

\section{Material e métodos}

O estudo foi realizado no Centro de Pesquisas e Conservação da Natureza (CPCN) Pró-Mata PUCRS, município de São Francisco de Paula, região nordeste do Estado do Rio Grande do Sul (RS), em uma área de 78 ha, com predominância de vegetação campestre circundada por florestas. A altitude é de aproximadamente $912 \mathrm{~m}$. A temperatura média anual em São Francisco de Paula é de $14,5^{\circ} \mathrm{C}$, com médias de temperaturas máximas e mínimas de $20,3{ }^{\circ} \mathrm{C}$ e $9,9^{\circ} \mathrm{C}$, respectivamente. A região apresenta altos índices pluviométricos durante todo o ano, sendo a média anual de $2252 \mathrm{~mm}$ (Backes et al. 2000). O CPCN Pró-Mata abrange uma zona caracterizada pelo encontro de três formações vegetais importantes: os Campos de Cima da Serra e a Floresta Ombrófila Mista ou Floresta com Araucária nas áreas de maior altitude e a Floresta Ombrófila Densa ou Mata Atlântica nas regiões de encosta (Marchiori 2002). Uma das peculiaridades da área de estudo é a exclusão do fogo e do pastejo desde 1993 (Oliveira \& Pillar 2004). 
A chuva de sementes foi avaliada com o uso de coletores para retenção dos diásporos (frutos e sementes), confeccionados com canos de PVC, tela plástica com malha de $2 \mathrm{~mm}$, linha de nylon e arame galvanizado, cada coletor com área de $314 \mathrm{~cm}^{2}$ (Fig. 1). Os coletores foram posicionados a uma altura de $50 \mathrm{~cm}$ do solo, coincidindo com a altura média do estrato de gramíneas nas áreas de campo. Portanto, neste estudo não foi avaliada a dispersão de sementes em nível de solo, apenas aquelas dispersas por aves ou morcegos, as autocóricas e as anemocóricas. Os diásporos foram removidos dos coletores mensalmente entre dezembro de 2006 e junho de 2007. Esse período foi definido após uma revisão bibliográfica sobre a fenologia das espécies lenhosas locais, onde foi verificado que este seria o período onde a grande maioria das espécies tinha potencial de frutificação. O material coletado foi acondicionado em sacos de papel e posteriormente triado em laboratório. Para a identificação das sementes e frutos foram utilizados material da literatura, exsicatas do Herbário Instituto de Biociências (ICN) da Universidade Federal do Rio Grande do Sul e do Laboratório de Botânica do Museu de Ciências e Tecnologia Pontifícia Universidade Católica do Rio Grande do Sul (PUC-RS), assim como de exemplares coletados no CPCN Pró-Mata para confirmação da identificação das espécies. Com base nestas informações, as espécies foram caracterizadas quanto às síndromes de dispersão, conforme a morfologia dos frutos segundo Pijl (1982).

Embora tanto frutos quanto sementes fossem capturados nos coletores, para as análises dos dados foi utilizado o número estimado de sementes. Para tanto, para aquelas

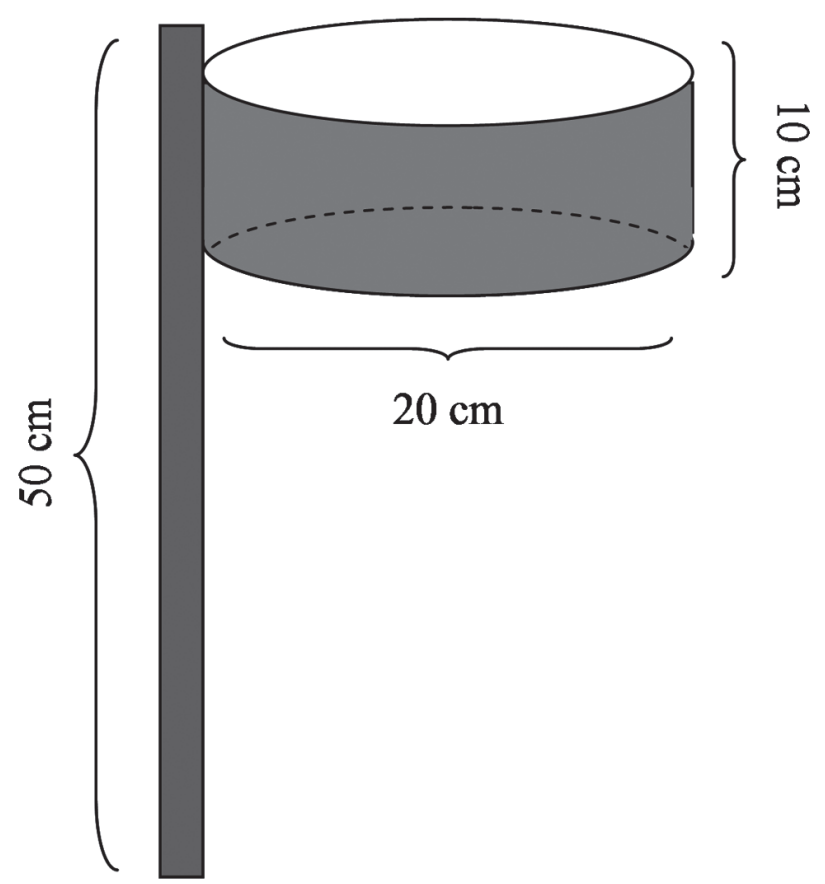

Figura 1. Esquema ilustrando o coletor de sementes utilizada nesse estudo no Centro de Pesquisas e Conservação da Natureza Pró-Mata PUC RS, São Francisco de Paula, RS. As dimensões estão descritas da figura. espécies cujos diásporos eram frutos, fez-se a estimativa a partir da multiplicação do número de frutos pelo número médio de sementes por fruto. Os valores médios de sementes por fruto foram obtidos com informações da literatura (Reitz 1969 a 1989) ou, quando não havia esta informação, pela contagem do número de sementes em 30 frutos de cada espécie. A precisão dessa estimativa foi avaliada por auto-reamostragem (método bootstrap, Pillar 1998) com o aplicativo computacional MULTIV (Pillar 2007). Quando possível, as sementes foram identificadas em nível de espécie, caso contrário foram mantidas em nível de gênero ou família, estas segundo delimitação de APG II (2003). Entretanto, todos os níveis taxonômicos das sementes foram doravante considerados espécies, para cálculos de riqueza e diversidade.

Os coletores foram instalados em seis tipos de ambiente (tratamentos) estruturalmente distintos, localizados na transição floresta-campo (borda) e sob indivíduos isolados (poleiros) na matriz campestre. Na transição floresta-campo, os tratamentos foram: floresta (FL; ca. 10 $\mathrm{m}$ da borda), borda (BO; limite dos indivíduos arbóreos da floresta contínua), campo com predomínio de formações arbustivas de B. uncinella (CB; ca. $10 \mathrm{~m}$ de $\mathrm{BO}$ ) e campo com predomínio de gramíneas (CG; $c a .10 \mathrm{~m}$ de BO). Como poleiros (indivíduos isolados no campo), foram selecionados 14 indivíduos de A. angustifolia (AA) e 14 de B. uncinella (BU), por serem importantes no processo de expansão florestal em áreas de campo excluído no planalto sul-brasileiro (Oliveira \& Pillar 2004; Duarte et al. 2006a; Dos Santos \& Pillar 2007; Duarte et al. 2010). Como critério de isolamento, considerou-se a ausência de qualquer outro indivíduo lenhoso inserido na matriz campestre num raio mínimo de $20 \mathrm{~m}$ e localizados a uma distância máxima de $100 \mathrm{~m}$ da borda florestal mais próxima. Determinou-se esta distância mínima entre os poleiros, pois foi considerado que a partir desta distância os poleiros seriam independentes quanto à chuva de sementes.

Ao longo da transição floresta-campo foram selecionados 12 locais de amostragem no ambiente floresta (FL), 12 locais no ambiente borda (BO) e 12 no ambiente campo (6 no ambiente CB e 6 no ambiente CG). Em cada local de amostragem foram colocadas três unidades amostrais (UA), sendo cada unidade amostral composta por três pares de coletores (total de $0,188 \mathrm{~m}^{2}$ por UA). Assim, os tratamentos CB e CG tiveram seis unidades amostrais cada $\left(1,128 \mathrm{~m}^{2}\right.$ amostrados por tratamento), enquanto FL e BO foram representados por 12 cada $\left(2,256 \mathrm{~m}^{2}\right)$. Os coletores de sementes foram instalados sempre dispostos em uma reta perpendicular a linha de borda florestal, com $10 \mathrm{~m}$ de distância entre estas retas, utilizando como referencial a linha de borda florestal.

O número de coletores instalados sob cada poleiro seguiu o critério de dois coletores a cada $10 \mathrm{~m}^{2}$ de área de copa. A média de coletores sob os indivíduos de $A$. angustifolia foi seis. $B$. Uncinella não apresentou área 
de copa superior a $10 \mathrm{~m}^{2}$, portanto foram dois coletores por indivíduo totalizando $5,26 \mathrm{~m}^{2}$ de área amostral. Cada indivíduo poleiro foi considerado uma unidade amostral, sendo somadas as abundâncias de sementes de todos os coletores sob cada indivíduo.

Os dados obtidos de cada unidade amostral serviram de base para a descrição da comunidade de espécies florestais lenhosas dispersadas para as áreas campestres. Para as análises foi utilizado o somatório de sementes de cada espécie coletadas durante os sete meses de amostragem em cada unidade amostral. Foram testadas, usando análises de variância com teste de aleatorização (Pillar \& Orlóci 1996), as hipóteses de independência entre os tratamentos (FL, $\mathrm{BO}, \mathrm{CB}, \mathrm{CG}, \mathrm{AA}$ e $\mathrm{BU}$ ) quanto à composição de espécies, densidade total de sementes (número de sementes por $\mathrm{m}^{2}$ de área total de coletores por unidade amostral) e proporção de espécies zoocóricas e não zoocóricas.

A análise de variância foi multivariada (MANOVA) quando os tratamentos foram comparados quanto à composição de espécies de sementes e univariada (ANOVA) quando o foram quanto à densidade total de sementes e a riqueza de espécies. Os testes de aleatorização usaram somas de quadrados computadas a partir de distâncias euclidianas (ANOVA) e de distância de corda (MANOVA) e as probabilidades em cada teste foram geradas com base em 10.000 permutações aleatórias (Pillar \& Orlóci 1996). Sempre que a hipótese de independência entre tratamentos foi rejeitada, contrastes foram avaliados via testes de aleatorização para identificar quais tratamentos diferiram estatisticamente (Pillar \& Orlóci 1996). Previamente às análises, os dados foram transformados por raiz quadrada (Podani 2000), exceto nos testes em que foi avaliada a riqueza de espécies, em que os dados não foram transformados. Também foi utilizado método de ordenação para avaliar a composição de espécies, através de Análise de Coordenadas Principais, tendo como medida de semelhança distância de corda entre unidades amostrais. Todas as análises foram realizadas no programa de estatística MULTIV (Pillar 2007).

\section{Resultados}

Do total de 3.505 sementes coletadas, foram encontradas 18 táxons de sementes, dos quais $82,4 \%$ apresentaram síndrome zoocórica e 17,6\% não zoocórica. Os tratamentos FL e BO foram caracterizados por uma alta densidade de sementes, com $70 \%$ e $23 \%$ do total, respectivamente; o tratamento AA apresentou 6\%, enquanto que CB, BU e CG apresentaram menos de $1 \%$ do total de sementes: 0,65\%, $0,25 \%$ e $0,1 \%$ respectivamente. Em relação à composição de espécies, houve diferença significativa entre os tratamentos, exceto: AA e CB ( $p=0.424)$, BU e CG ( $p=0.677), B O$ e CB $(\mathrm{p}=0.204)$ e CG e CB $(\mathrm{p}=0,241)$. O padrão de distribuição das espécies de sementes conforme os tratamentos pode ser visualizado no diagrama de dispersão obtido pela análise de ordenação (Fig. 2). Sementes de Croton sp. predominam nas unidades amostrais de borda, ao passo que Myrsine lorentziana caracteriza áreas sob poleiros de Araucária e as áreas na floresta tipicamente apresentam mais espécies simultaneamente.

Quanto à densidade total de sementes, apenas os tratamentos AA e CB $(\mathrm{p}=0,47)$ e BU e CG $(\mathrm{p}=1)$ tiveram abundâncias semelhantes (Fig. 3A). Quanto à riqueza de táxons nas áreas avaliadas, repetiu-se o padrão encontrado na análise de densidade total de sementes, ou seja, os contrastes AA-CB e BU-CG não apresentaram diferenças significativas ( $p=0,518 \mathrm{e} p=1$, respectivamente) (Fig. 3B).

Considerando apenas as espécies zoocóricas quanto à densidade total de sementes, os tratamentos que tiveram densidades semelhantes foram AA e CB $(\mathrm{p}=0,149)$ e BU e $\mathrm{CG}(\mathrm{p}=1)$, enquanto que $\mathrm{BO}$ e AA foram significativamente diferentes $(\mathrm{p}=0,0002)$ (Fig. 4A). Quanto à riqueza de espécies, os tratamentos AA e BO, AA e CB, BU e CG, e BO e $\mathrm{CB}$ tiveram valores que não diferiram estatisticamente (Fig. $4 \mathrm{~B})$. Por outro lado, tanto a densidade quanto a riqueza de espécies não zoocóricas foram semelhantes para os tratamentos AA-BU e BU-CG ( $\mathrm{p}=1)$, AA e BU $(\mathrm{p}=0,30)$ e FL e $\mathrm{BO}(\mathrm{p}=0,87)$ (Fig. $5 \mathrm{~A}$ e $5 \mathrm{~B})$.

\section{Discussão}

O processo de dispersão zoocórica também se mostrou predominante nos subtrópicos em áreas de encontro entre Floresta com Araucária e Campos, como já descrito para regiões tropicais (Howe \& Smallwood 1982; Willson et al. 1989). Nas áreas de campo, indivíduos isolados de $A$. angustifolia e as formações arbustivas da $B$. uncinella apresentaram maior chuva de sementes do que áreas de campo limpo (sem arbustos altos) e sob indivíduos de B. uncinella isolados, corroborando com outros estudos que também verificaram que estruturas mais proeminentes em áreas de campo incrementam a chuva de sementes (Fuentes et al. 1984; Holl 1998; Duncan \& Chapman 1999; Shiels \& Walker 2003). Os resultados diferenciados entre indivíduos isolados de A. angustifolia e B. uncinella quanto à densidade de sementes podem ser relacionados às diferenças de altura $\mathrm{e}$ estrutura de copa destas espécies, visto que a A. angustifolia é um poleiro de maior porte, podendo acarretar diferenças de uso preferencial da avifauna. Estas diferenças também foram encontradas em pastagens na África por Duncan \& Chapman (1999), em que a deposição de sementes em áreas campestres foi proporcional à altura das árvores isoladas.

Em relação à composição de espécies, apesar de indivíduos de B. uncinella apresentarem mais sementes depositadas do que as áreas de campo, as diferenças entre estes tratamentos não foram significativas, sendo este poleiro pouco representativo para o recrutamento de sementes. Já os indivíduos de A. angustifolia foram semelhantes às formações arbustivas de B. uncinella, que por sua vez foram semelhantes às áreas de bordas florestais. Isto sugere que as formações arbustivas e indivíduos de $A$. angustifolia comportam-se como extensões 


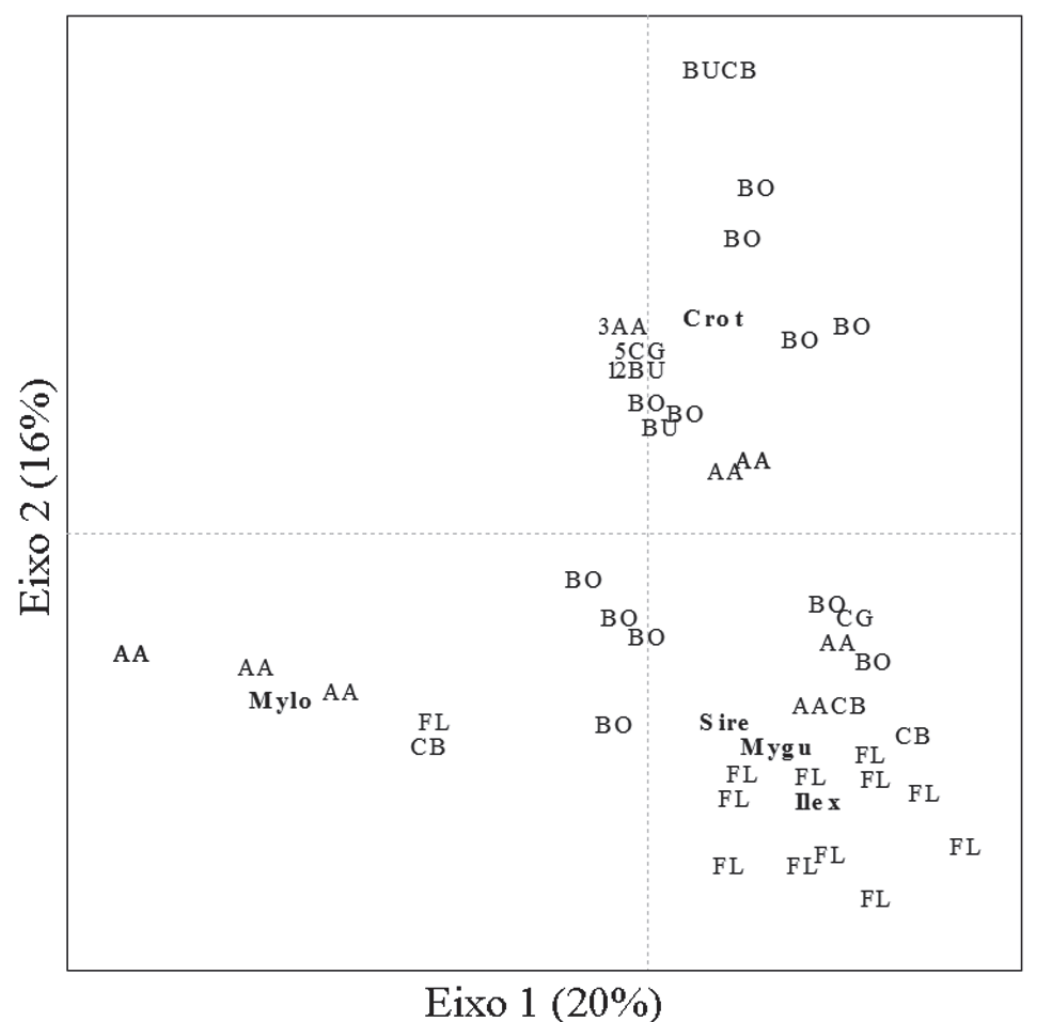

\begin{abstract}
Figura 2. Diagrama de ordenação da composição de espécies de sementes amostradas em coletores no Centro de Pesquisas e Conservação da Natureza Pró-Mata PUC RS. Foi utilizada análise de coordenadas principais com distância de corda entre unidades amostrais com as variáveis transformadas por raiz quadrada. A percentagem de variação contida em cada eixo e as espécies que apresentaram correlação com pelo menos um dos eixos igual ou acima de $0,5(r=|0.5|)$ estão indicadas. FL (floresta, $\mathrm{n}=12), \mathrm{BO}$ (borda florestal, $\mathrm{n}=12$ ), CB (campo com predomínio de $B$. uncinella, $\mathrm{n}=6$ ), $\mathrm{CG}$ (campo com predomínio de gramíneas, $\mathrm{n}=6), \mathrm{BU}$ (B. uncinella, $\mathrm{n}=14$ ) e AA (A. angustifolia, $\mathrm{n}=14$ ). Mylo (Myrsine lorentziana), Crot (Croton sp.), Sire (Siphoneugena reitzii), Myob (Myrcia guianensis), Ilex (Ilex spp.).
\end{abstract}

das bordas florestais sobre as áreas campestres, o que poderia acelerar os processos sucessionais de áreas florestais sobre os campos. Estes resultados da chuva de sementes corroboram as idéias de Klein (1960) de que a expansão da Floresta com Araucária sobre os campos ocorre aos saltos, através de indivíduos de A. angustifolia na matriz campestre e também pela expansão das bordas florestais, como observado por Duarte et al. (2006), onde foi avaliada a presença de plântulas associadas a indivíduos isolados de $A$. angustifolia, e verificado por Oliveira \& Pillar (2004), onde áreas de formações arbustivas de B. uncinella foram mais favoráveis à expansão da Floresta com Araucária sobre os campos. Quanto à densidade total de sementes e riqueza de espécies, houve um gradiente de transição de áreas florestais para áreas campestres, tendo as áreas florestais valores maiores, como também encontrado por Holl (2000) na Costa Rica, e possuindo valores intermediários para árvores de $A$. angustifolia e formações arbustivas de $B$. uncinella. Isto sugere que possivelmente há também um gradiente de fauna dispersora relacionados com a estrutura da vegetação nestas áreas de transição floresta-campo. Segundo De Steven (1991), enquanto a chuva de sementes para áreas de campo abandonadas depende da composição da comunidade próxima (área-fonte), outras diferenças intrínsecas entre as espécies podem afetar a chegada de sementes das mesmas, como uma fauna diferenciada de dispersores.

As análises quanto às síndromes de dispersão reforçam que a dispersão zoocórica é o principal guia nos processos de expansão florestal nestas áreas de ecótonos, visto que em relação a espécies com dispersão não zoocórica, não há sítios preferenciais de deposição de sementes nas áreas campestres, exceto nas formações arbustivas de B. uncinella, em que o recrutamento destas sementes foi maior depois de áreas de bordas e de floresta. Essas formações podem estar funcionando como anteparo para estas sementes anemocóricas, pois são formações mais densas do que árvores ou arbustos isolados no campo. Padrão semelhante também foi encontrado no Chile por Fuentes et al. (1984) em que formações arbustivas recrutaram mais sementes não zoocóricas em áreas campestres.

Visto que poleiros, assim como formações arbustivas em áreas campestres incrementam a chuva de sementes e estariam acelerando processos de sucessão vegetal, os resultados têm implicações principalmente para questões de manejo e conservação. Primeiro com relação à restauração 


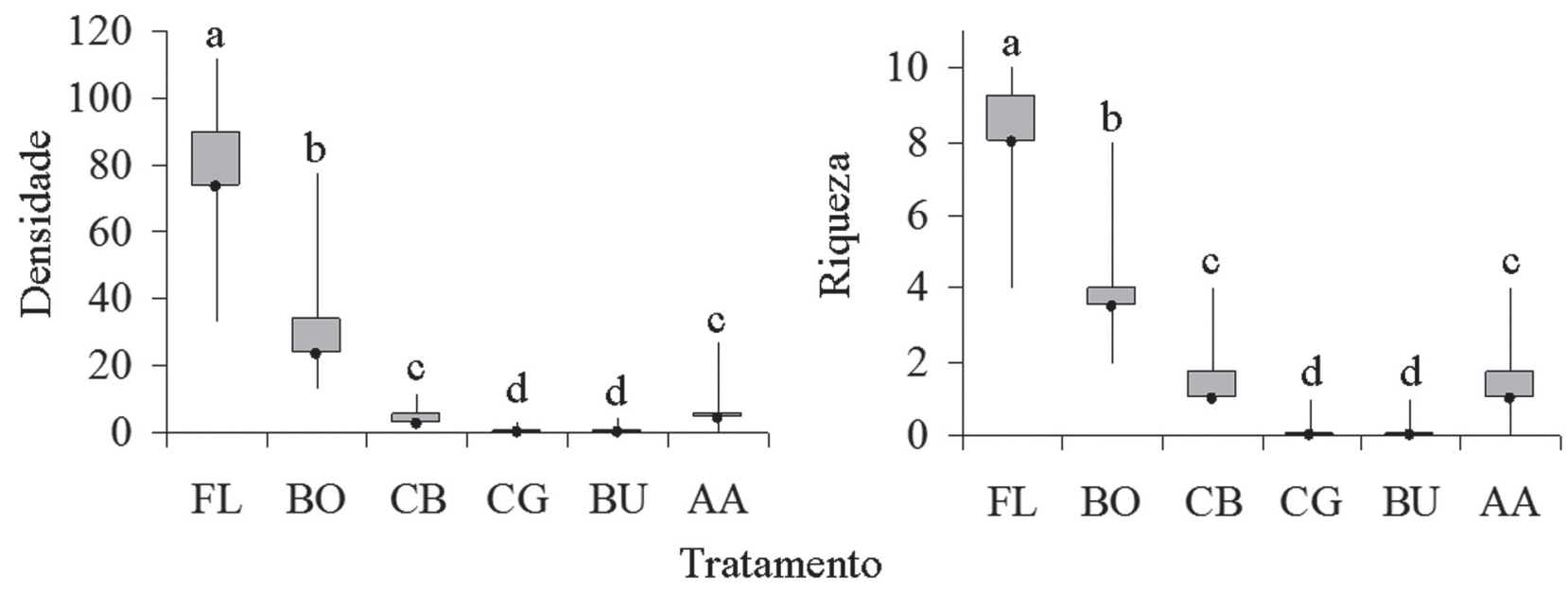

Figura 3. Densidade total de sementes (A) e riqueza de espécies (B) em cada tratamento amostradas em coletores no Centro de Pesquisas e Conservação da Natureza Pró-Mata PUC RS. Tratamentos seguidos da mesma letra não diferiram estatisticamente em contrastes pareados usando testes de aleatorização ( $\alpha>0,1)$. FL (floresta, $\mathrm{n}=12$ ), BO (borda florestal, $\mathrm{n}=12$ ), CB (campo com predomínio de B. uncinella, $\mathrm{n}=6$ ), CG (campo com predomínio de gramíneas, $\mathrm{n}=6$ ), BU (B. uncinella, $\mathrm{n}=14$ ) e AA (A. angustifolia, $\mathrm{n}=14$ ). O ponto no interior das caixas representa a mediana, as caixas representam o primeiro e o terceiro quartil e as linhas verticais representam a amplitude.

A

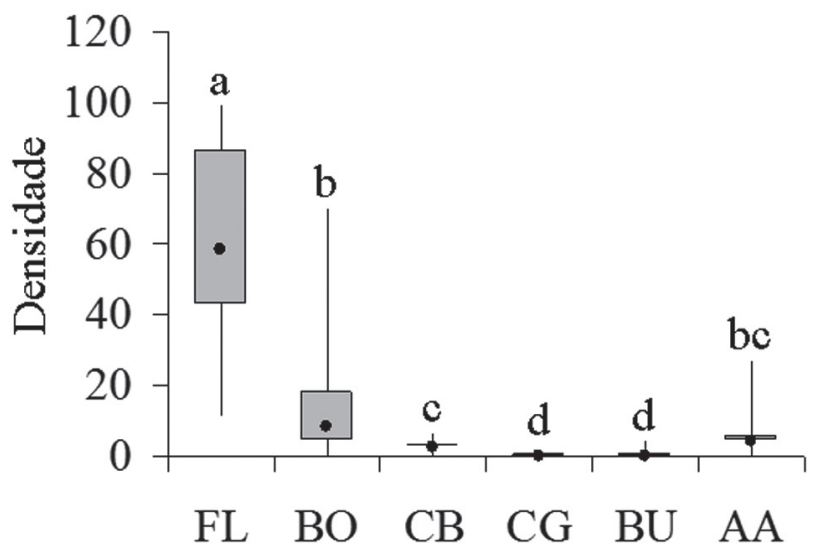

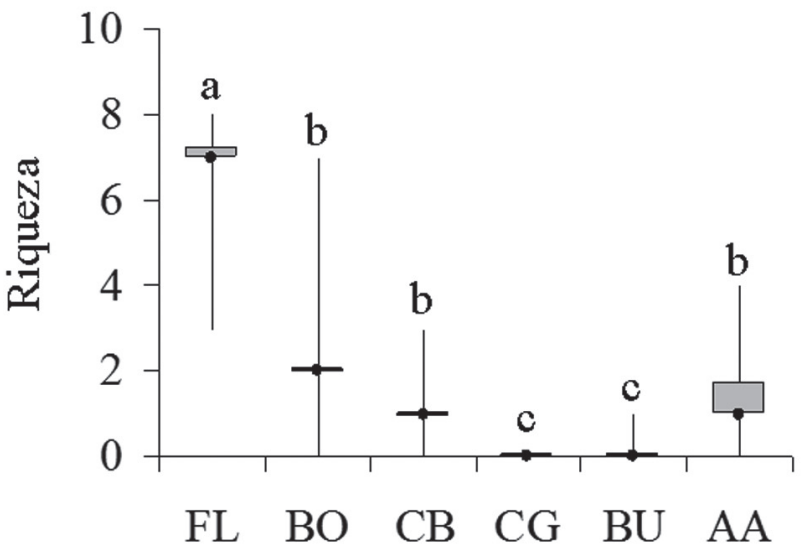

Tratamento

Figura 4. Densidade total de sementes de espécies zoocóricas (A) e riqueza de espécies zoocóricas (B) em cada tratamento amostradas em coletores no Centro de Pesquisas e Conservação da Natureza Pró-Mata PUC RS. Tratamentos seguidos da mesma letra não diferiram estatisticamente em contrastes pareados usando testes de aleatorização $(\alpha>0,1)$. FL (floresta, $\mathrm{n}=12$ ), BO (borda florestal, $\mathrm{n}=12$ ), CB (campo com predomínio de $B$. uncinella, $\mathrm{n}=6$ ), CG (campo com predomínio de gramíneas, $\mathrm{n}=6)$, BU (B. uncinella, $\mathrm{n}=14$ ) e AA (A. angustifolia, $\mathrm{n}=14$ ). O ponto no interior das caixas representa a mediana, as caixas representam o primeiro e o terceiro quartil e as linhas verticais representam a amplitude.

de áreas degradadas, indicando que o plantio de árvores ou a instalação de estruturas artificiais tendem a ser efetivos para recrutar sementes de espécies florestais. Segundo, trata dos subsídios científicos de que o manejo de formações campestres naturais na região de estudo, através da limitação de estruturas de vegetação diferenciada sobre as áreas abertas, por exemplo, é necessário à conservação deste bioma ameaçado e das espécies a ele associadas. Por outro lado, a terceira implicação deste trabalho é com relação ao processo de expansão florestal em si e à conservação do mesmo na ausência de qualquer intervenção humana nas áreas campestres. Portanto, a constatação de que a chuva de sementes de espécies florestais ocorre e apresenta padrões específicos conforme a estrutura da vegetação, apontando para o forte potencial de expansão da Floresta com Araucária sobre os campos, permite a pesquisadores e tomadores de decisões que ponderem sobre o que conservar, como manejar ou ainda como restaurar sistemas florestais. 


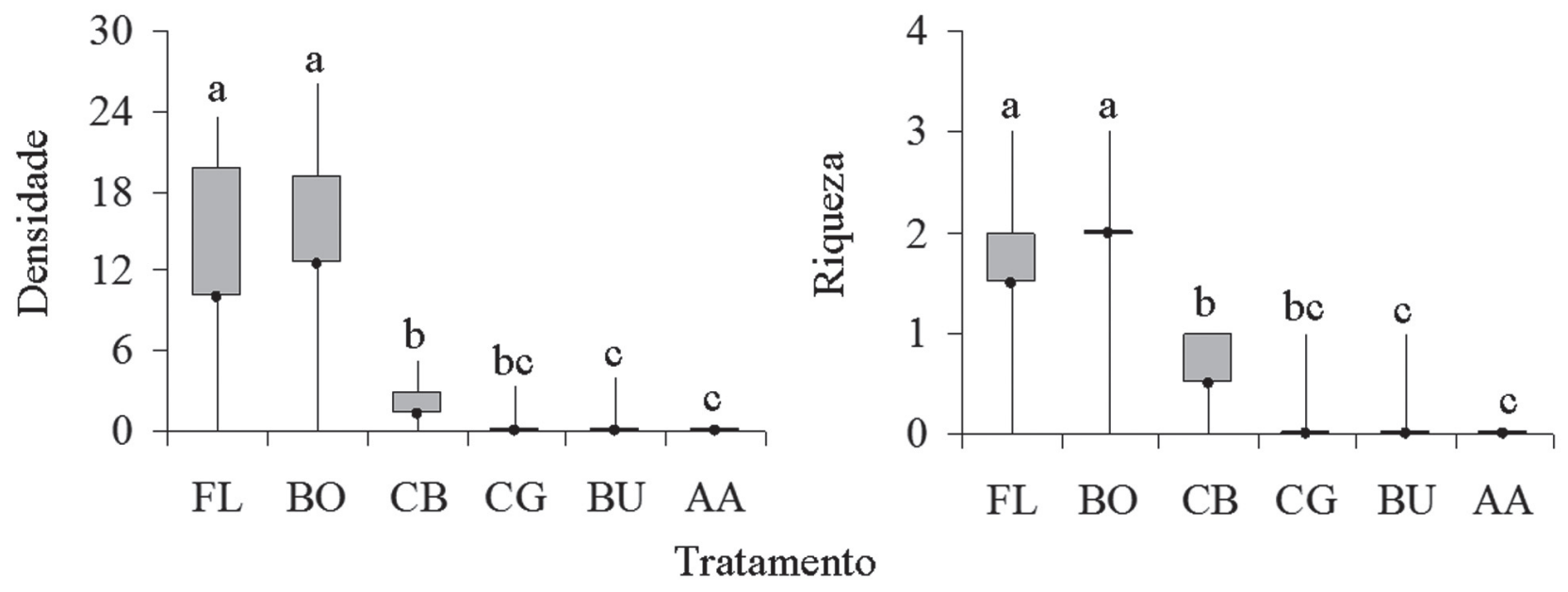

Figura 5. Densidade total de sementes de espécies não zoocóricas (A) e riqueza de espécies não zoocóricas (B) em cada tratamento amostradas em coletores no Centro de Pesquisas e Conservação da Natureza Pró-Mata PUC RS.. Tratamentos seguidos da mesma letra não diferiram estatisticamente em contrastes pareados usando testes de aleatorização $(\alpha>0,1)$. FL (floresta, $n=12$ ), BO (borda florestal, $n=12$ ), CB (campo com predomínio de B. uncinella, $n=6$ ), CG (campo com predomínio de gramíneas, $n=6$ ), BU (B. uncinella, $n=14$ ) e AA (A. angustifolia, $n=14$ ). O ponto no interior das caixas representa a mediana, as caixas representam o primeiro e o terceiro quartil e as linhas verticais representam a amplitude.

\section{Agradecimentos}

Ao Inter-American Institute for Global Change Research (IAI) pelo financiamento do projeto, ao Instituto do Meio Ambiente da PUCRS e ao Centro de Pesquisas e Conservação da Natureza Pró-Mata PUCRS pela autorização deste trabalho e por todo o apoio logístico prestado.

\section{Referências bibliográficas}

APG II. 2003. An update of the Angiosperm Phylogeny Group classification for the orders and families of flowering plants: APG II. Botanical Journal of the Linnean Society 141(4): 399-436.

Backes, A.; Fernandes, A.V. \& Zeni, D.J. (2000). Produção de folhedo em uma floresta com Araucaria angustifolia no sul do Brasil. Pesquisas (Botânica) 50: 97-117.

Behling, H.; Pillar, V.D.; Orlóci, L. \& Bauermann. S.G. 2004. Late Quaternary Araucaria forest, grassland (Campos), fire and climate dynamics, studied by high-resolution pollen, charcoal and multivariate analysis of Cambará do Sul core in southern Brazil. Palaeogeography, Palaeoclimatology, Palaeoecology 203: 277-297.

Calaway, R.M. \& Walker, L.R. 1997. Competition and facilitation: a synthetic approach to interactions in plant communities. Ecology 78(7): 1958-1965.

Caldato, S.L.; Floss, P.A.; Da Croce, D.M. \& Longhi, S.J. 1996. Estudo da regeneração natural, banco de sementes e chuva de sementes na Reserva Genética Florestal de Caçador, SC. Ciência Florestal 6(1): 27-38.

Clark, C.J.; Poulsen, J.R.; Connor, E.F.; Parker, V.T. 2004. Fruiting trees as dispersal foci in a semi-deciduous tropical forest. Oecologia 139: 66-75.

De Steven, D.1991. Experiments on mechanisms of tree establishment in old-field succession: seedling survival and growth. Ecology 72(3): 1076-1088.

Dos Santos, M.M.G. \& Pillar, V.D. 2007. Influência de poleiros naturais e artificiais na expansão da Floresta com Araucária sobre os campos, em São Francisco de Paula, RS. Revista Brasileira de Biociências 5(1): 594-596.

Duarte, L. da S.; Dos Santos, M.M.G.; Hartz, S.M. \& Pillar, V.D. $2006 a$. Role of nurse plants in Araucaria Forest expansion over grassland in south Brazil. Austral Ecology 31: 520-528.

Duarte, L.S.; Hofmann, G.S.; Dos Santos, M.M.G.; Hartz, S.M.; Pillar, V.D. 2010. Testing for the influence of niche and neutral factors on sapling community assembly beneath isolated woody plants in grasslands. Journal of Vegetation Science 21: 462-471.

Duarte, L. da S.; Machado, R.E.; Hartz, S.M. \& Pillar, V.D. 2006b. What saplings can tell us about Forest expansion over natural grasslands. Journal of Vegetation Science 17: 799-808.

Duncan, R.S. \& Chapman, C.A. 1999. Seed dispersal and potential Forest succession in abandoned agriculture in tropical Africa. Ecological Applications 9(3): 998-1008.

Fontoura, S.B.; Ganade, G. \& Larocca, J. 2006. Changes in plant community diversity and composition across edge between Araucaria Forest and pasture in South Brazil. Revista Brasileira de Botânica 29(1): 79-91.

Fuentes, E.R.; Otaiza, R.D.; Alliende, M.C.; Hoffmann, A. \& Poiani, A. 1984. Shrub clumps of the Chilean matorral vegetation - structure and possible maintenance mechanisms. Oecologia 62: 405-411.

Gómez-Aparicio, L.; Gómez, J.M.; Zamora, R. \& Boettinger, J.L. 2005. Canopy vs. soil effects of shrubs facilitating tree seedlings in Mediterranean montane ecosystems. Journal of Vegetation Science 16: 191-198.

Guevara, S.; Meave, J.; Moreno-Cassola, P. \& Laborde, J. 1992. Floristic composition and structure of vegetation under isolated tress in neotropical pastures. Journal of Vegetation Science 3: 655-664.

Holl, K.D.; Loik, M.E.; Lin, E.H.V. \& Samuels, I.A. 2000. Tropical montane Forest restoration in Costa Rica: overcoming barriers to dispersal and establishment. Restoration Ecology 8(4): 339-349.

Holl, K.D. 1998. Do bird perching structure elevate seed rain and seedling establishment in abandoned tropical pasture? Restoration Ecology 6: 253-261.

Holmgren, M.; Scheffer, M. \& Huston, M. A. 1997. The interplay of facilitation and competition in plant communities. Ecology 78(7): 1966-1975.

Howe, H.F. \& Smallwood, J. (1982). Ecology of seed dispersal. Annual Review of Ecology and Systematics 13: 201-228.

Klein, R.M. 1960. O aspecto dinâmico do pinheiro brasileiro. Sellowia 12: $17-44$. 
Machado, R.E. 2004. Padrões vegetacionais em capões de floresta com araucária no planalto nordeste do Rio Grande do Sul, Brasil. MSC. Dissertação, Porto Alegre, UFRGS.

Marchiori, J.N.C. 2002. Fitogeografia do Rio Grande do Sul: enfoque histórico e sistemas de classificação. Porto Alegre, EST Edições.

Nepstad, D.C.; Uhl, C.; Pereira, C.A. \& Silva, J.M.C. 1996. A comparative study of tree establishment in abandoned pasture and mature forest of eastern Amazonia. Oikos 76: 25-39.

Oliveira, J.M. \& V.D. Pillar. 2004. Vegetation dynamics on mosaics of Campos and Araucaria forest between 1974 and 1999 in Southern Brazil. Community Ecology 5(2): 197-202.

Overbeck, G.E.; Müller, S.C.; Fidelis, A.; Pfadenhauer, J.; Pillar, V.D.; Blanco, C.C.; Boldrini, I.I.; Both, R. \& Forneck, E.D. 2007. Brazil's neglectec biome: the South Brazilian Campos. Perspectives in Plant Ecology, Evolution and Systematics 9: 101-116.

Peterson, C.J. \& Haines, B.L. 2000. Early succesional patterns and potential facilitacion of woody plant colonization by rotting logs in Premontane Costa Rican pastures. Restoration Ecology 8(4): 361-369.

Pijl, L. van der. 1982. Principals of dispersal in higher plants. New York, Springer-Velag.

Pillar, V.D. 1998. Sampling sufficiency in ecological surveys. Abstracta Botanica 22: 37-48.

Pillar, V.D. 2003. Dinâmica da expansão florestal em mosaicos de floresta e campos no sul do Brasil. Pp. 209-216. In: Claudino-Sales, V. (Ed.) Ecossistemas Brasileiros: Manejo e Conservação. Fortaleza, Expressão Gráfica.

Pillar, V.D. 2007. MULTIV, software para análise multivariada, testes de hipóteses e auto-reamostragens. Porto Alegre, Departamento de Ecologia, UFRGS (versions 2.5b for Machintosh and Windows available at: http://ecoqua.ecologia.ufrgs.br).

Pillar, V.D. \& L. Orlóci. 1996. On randomization testing in vegetation science: multifactor comparisons of relevé groups. Journal of Vegetation Science 7: 585-592.

Podani, J. 2000. Multivariate data analysis in Ecology and Systematics. The Hauge, SPB Academic Publishing.

Rambo, B. 1956. A fisionomia do Rio Grande do Sul. 2 ed. Porto Alegre, Editora Selbach \& Cia.

Reitz, R. 1965-1989. Flora Ilustrada Catarinense. Itajaí, Herbário Barbosa Rodriguez.

Shiels, A.B. \& Walker, L.R. 2003. Bird perches increase forest seeds on Puerto Rican landslides. Bird perches increase forest seeds on Puerto Rican landslides. Restoration Ecology 11(4): 457-465.

Válio, I.F.M. \& Scarpa, F.M. 2001. Germination of seeds of tropical pioneer species under controlled and natural conditions. Revista brasileira de Botânica 24(1): 79-84.

Waechter, J.L.; Cestaro, L.A. \& Miotto, S.T.S. 1984. Vegetation types in the Ecological Station of Aracuri, Esmeralda, Rio Grande do Sul, Brazil. Phytocoenologia 12(2,3): 261-269.

Willson, M.F.; Irvine, A.K. \& Walsh, N.G. 1989. Vertebrate dispersal syndromes in some Australian and New Zealand plant communities, with geographic comparisons. Biotropica 21(2): 133-147.

Zanini, L. \& Ganade, G. 2005. Restoration of Araucaria Forest: the role of perches, pioneer vegetation, and soil fertility. Restoration Ecology 13(3): 507-514.

Zanini, L.; Ganade, G. \& Hübel, I. 2006. Facilitation and competition influence succession in subtropical old field. Plant Ecology 185: 179-190. 